\title{
Two-color discrete localized modes and resonant scattering in arrays of nonlinear quadratic optical waveguides
}

\author{
Mario I. Molina, ${ }^{1}$ Rodrigo A. Vicencio, ${ }^{2}$ and Yuri S. Kivshar ${ }^{3}$ \\ ${ }^{1}$ Departamento de Física, Facultad de Ciencias, Universidad de Chile, Casilla 653, Santiago, Chile \\ ${ }^{2}$ Max-Planck-Insitut für Physik komplexer Systeme, Nöthnitzerstrasse 38, Dresden 01187, Germany \\ ${ }^{3}$ Nonlinear Physics Center, Research School of Physical Sciences and Engineering, Australian National University, \\ Canberra ACT 0200, Australia \\ (Received 17 March 2005; published 30 September 2005)
}

\begin{abstract}
We analyze the properties and stability of two-color discrete localized modes in arrays of channel waveguides where tunable quadratic nonlinearity is introduced as a nonlinear defect by periodic poling of a single waveguide in the array. We show that, depending on the value of the phase mismatch and the input power, such two-color defect modes can be realized in three different localized states. We also study resonant light scattering in the arrays with the defect waveguide.
\end{abstract}

DOI: 10.1103/PhysRevE.72.036622

PACS number(s): 42.81.Qb, 05.45.-a, 42.65.-k

\section{INTRODUCTION}

The study of nonlinear propagation of light in periodic photonic structures recently attracted strong interest due to the unique possibility of observing experimentally an interplay between the effects of nonlinearity and periodicity, including the generation of spatial optical solitons [1]. Recently fabricated nonlinear periodic structures such as arrays of weakly coupled nonlinear optical waveguides can support distinctive types of self-trapped optical modes [2-5]. Moreover, linear properties such as diffraction may differ dramatically compared to those in the corresponding continuous systems.

In addition to cubic nonlinear periodic systems, during last years a growing interest is observed in the study of nonlinear optics associated with the so-called "quadratic nonlinearities" which may produce the effects resembling those known to occur in cubic nonlinear materials. Typical examples are all-optical switching phenomena in interferometric or coupler configurations as well as the formation of spatial and temporal solitons in planar waveguides [6]. One of the recent highlights in this field is the first experimental demonstration of discrete solitons with two frequency components mutually locked by a quadratic nonlinearity [7]. These optical experiments have been performed in arrays of weakly coupled channel waveguides with tunable cascaded quadratic nonlinearity, and they demonstrated a good agreement with the theoretical analysis [8]. As a matter of fact, it was demonstrated that arrays of coupled channel waveguides fabricated in a periodically poled Lithium Niobate slab represent a convenient system to verify experimentally many theoretical predictions. These experimental observations open novel perspectives for employing larger nonlinearities in the periodic systems with quadratic materials.

In this paper, we analyze the properties and stability of two-color discrete localized modes in arrays of channel waveguides. In particular, we show that when periodic poling is applied to just a single waveguide in the array, it creates a nonlinear defect [9-11] that may support strongly localized discrete modes similar to discrete solitons and also display specific resonant scattering properties.

The paper is organized as follows. In Sec. II we introduce our model and find the profiles of two-color discrete localized modes. We show that such modes can exist in three different states, and they can be observed in the same array depending on the array parameters and the value of the input power. Section III is devoted to the study of the resonant light transmission through the defect waveguide that can be associated with the Fano resonance. Finally, Sec. IV concludes the paper.

\section{TWO-COLOR LOCALIZED MODES}

Being motivated by the design of the periodic photonic structures recently employed for experiments [7], we consider an array of weakly coupled linear waveguides where one waveguide has periodic poling, and therefore it possesses a quadratic nonlinear response. When the matching conditions are satisfied, the fundamental-frequency (FF) mode with the frequency $\omega$ generates the second-harmonic $(\mathrm{SH})$ wave at the frequency $2 \omega$, so that such a structure with the poled waveguide may behave as a nonlinear defect with localized quadratic nonlinearity.

In the tight-binding approximation [5], the effective equations for the complex envelopes of the FF wave $\left(u_{n}\right)$ and its SH component $\left(v_{n}\right)$ coupled at the defect waveguide (at $n$ $=0$ ) can be written in the form

$$
\begin{gathered}
i \frac{d u_{n}}{d z}+c_{u}\left(u_{n+1}+u_{n-1}\right)+2 u_{0}^{*} v_{0} \delta_{n 0}=0, \\
i \frac{d v_{n}}{d z}+c_{v}\left(v_{n+1}+v_{n-1}\right)-\Delta v_{n}+u_{0}^{2} \delta_{n 0}=0,
\end{gathered}
$$

where $c_{u}$ and $c_{v}$ are the coupling coefficients, $\Delta$ is the phase mismatch parameter, and $\delta_{n 0}$ is a delta function.

The discrete model (1) has two conserved quantities, the total power 


$$
P=\sum_{n=-\infty}^{\infty}\left(\left|u_{n}\right|^{2}+2\left|v_{n}\right|^{2}\right)
$$

and the Hamiltonian. We look for stationary solutions of the coupled equations in the form

$$
u_{n}(z)=u_{n} e^{i \lambda z}, \quad v_{n}(z)=v_{n} e^{2 i \lambda z},
$$

where we assume, without loss of generality, that the amplitudes $u_{n}$ and $v_{n}$ are real. As a result, Eqs. (1) become

$$
\begin{gathered}
\lambda u_{n}=c_{u}\left(u_{n+1}+u_{n-1}\right)+2 u_{0} v_{0} \delta_{n 0}, \\
2 \lambda v_{n}=c_{v}\left(v_{n+1}+v_{n-1}\right)-\Delta v_{n}+u_{0}^{2} \delta_{n 0} .
\end{gathered}
$$

We look for spatially localized discrete states in the form $u_{n}=u_{0} \eta^{|n|}$ and $v_{n}=v_{0} \xi^{|n|}$, where $|\eta|,|\xi|<1$, and obtain from Eqs. (3) at $n \neq 0$ two relations, $\lambda=c_{u}\left(\eta+\eta^{-1}\right)$ and $2 \lambda=c_{v}(\xi$ $\left.+\xi^{-1}\right)-\Delta$, which can be combined to yield

$$
c_{v}\left(\xi+\frac{1}{\xi}\right)-2 c_{u}\left(\eta+\frac{1}{\eta}\right)=\Delta .
$$

On the other hand, for the defect waveguide at $n=0$ we obtain $\lambda=2 c_{u} \eta+2 v_{0}$ and $2 \lambda v_{0}=2 c_{v} v_{0} \xi-\Delta v_{0}+u_{0}^{2}$. After some simple algebra, we solve for the parameters $u_{0}$ and $v_{0}$ in terms of $\eta$ and $\xi$ and obtain

$$
v_{0}=\frac{c_{u}}{2}\left(\frac{1}{\eta}-\eta\right), \quad u_{0}^{2}=c_{v} v_{0}\left(\frac{1}{\xi}-\xi\right) .
$$

In addition, Eq. (2) can be expressed as

$$
P=u_{0}^{2}\left(\frac{1+\eta^{2}}{1-\eta^{2}}\right)+2 v_{0}^{2}\left(\frac{1+\xi^{2}}{1-\xi^{2}}\right),
$$

and the next step is to replace the amplitudes $u_{0}$ and $v_{0}$ in Eq. (6) and obtain the second equation

$$
c_{u} c_{v} \frac{\left(1-\xi^{2}\right)}{2 \eta \xi}\left(1+\eta^{2}\right)+c_{u}^{2} \frac{\left(1-\eta^{2}\right)^{2}}{2 \eta^{2}} \frac{\left(1+\xi^{2}\right)}{\left(1-\xi^{2}\right)}=P .
$$

Equations (4) and (7) constitute a system of two coupled nonlinear equations for the functions $\eta$ and $\xi$. Moreover, Eq. (4) depends on the mismatch parameter $\Delta$, whereas Eq. (7) depends on the total power $P$.

First, we assume that $c_{u}, c_{v}>0$. From Eq. (5) we find that if $v_{0}>0$ then $0<\xi, \eta<1$. On the other hand, if $v_{0}<0$, then $-1<\xi, \eta<0$. Also, without loss of generality, we can consider only $u_{0}>0$. Thus, we should find real solutions for the values $\eta$ and $\xi$ in the domain defined by the conditions 0 $<\eta, \xi<1$ and $-1<\eta, \xi<0$ on the plane $(\eta, \xi)$.

From the structure of Eqs. (4) and (7) we notice that a change $\Delta \rightarrow-\Delta$ is equivalent to the change $(\eta, \xi) \rightarrow(-\eta$, $-\xi)$. Thus, we only need to consider positive values of $\Delta$. In order to find spatially localized states, we should analyze the functions of $\eta$ and $\xi$ defined by the left-hand sides of the expressions (4) and (7), for varying values of the mismatch parameter $\Delta$ and the total power $P$. This analysis reveals that, in general, for a given value of $\Delta$, a minimum value of the power is required to support a localized state at the defect.

As an example, in Figs. 1(a)-1(d) we show several profiles of the localized modes for the case $\Delta=0$ and two values
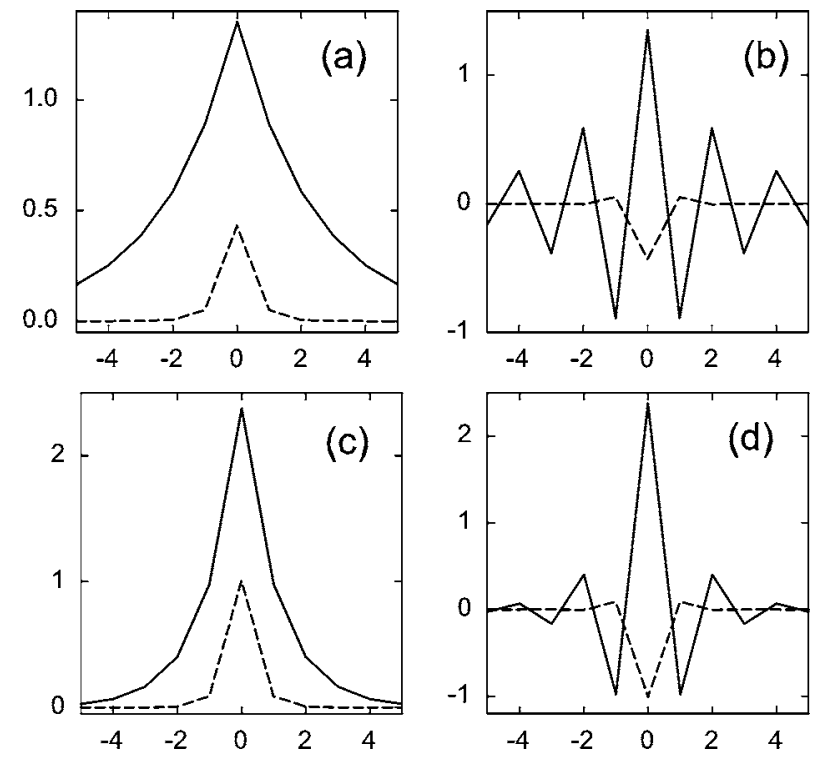

FIG. 1. Examples of localized modes at $\Delta=0$ and two values of the total power above the threshold: (a), (b) $P=5$ and (c), (d) $P$ $=10$. Solid and dashed curves show the fundamental-frequency $\left(u_{n}\right)$ and second-harmonic $\left(v_{n}\right)$ fields, respectively.

of the power $P$, for the typical values $c_{u}=1$ and $c_{v}=0.5$. Below a certain threshold power, there exist no localized states, but above the threshold two localized modes appear, unstaggered (a) and staggered (b) modes, respectively [see Figs. 1(a) and 1(b)]. As the total power $P$ increases, these two modes become more localized, as shown in Figs. 1(c) and $1(\mathrm{~d})$.

For a finite positive mismatch $\Delta$ and increasing power $P$, we find that there is a threshold power below which no localized states exist. When the power exceeds the first threshold, at least one (staggered) localized modes becomes possible and then the other mode appears for larger powers. As the value of the mismatch $\Delta$ increases further, it can be proven that the contour curve associated with Eq. (4), which depends on $\Delta$, experiences a curvature change at $\Delta=2\left(2 c_{u}\right.$ $\left.-c_{v}\right)=3$ and, as a result, a double root is possible at a certain power level. Further power increase gives rise to two staggered localized states. Even further increase in the power makes possible another (unstaggered) localized state to exist, as shown in Fig. 2 at $\Delta=5$.

The threshold value of the total power that corresponds to the appearance of the first localized mode can be found analytically in the form

$$
P_{\min }^{1}(\Delta)=c_{u}\left[\left(\Delta+4 c_{u}\right)^{2}-4 c_{v}^{2}\right]^{1 / 2},
$$

for the condition $\Delta>2\left(c_{v}-2 c_{u}\right)$, and

$$
P_{\min }^{2}(\Delta)=c_{u}\left[\left(\Delta-c_{u}\right)^{2}-4 c_{v}^{2}\right]^{1 / 2},
$$

for the condition $\Delta<2\left(2 c_{u}-c_{v}\right)$. In the first case, the lowest localized mode is unstaggered, whereas in the second case, it is staggered. Applying these results to Fig. 1, we obtain $P_{\text {min }}^{1}(0)=P_{\text {min }}^{2}(0)=\sqrt{15}=3.873$. By solving Eq. (1) numerically, we have checked the stability of all localized modes to propagation, and we came to the conclusion that such modes 

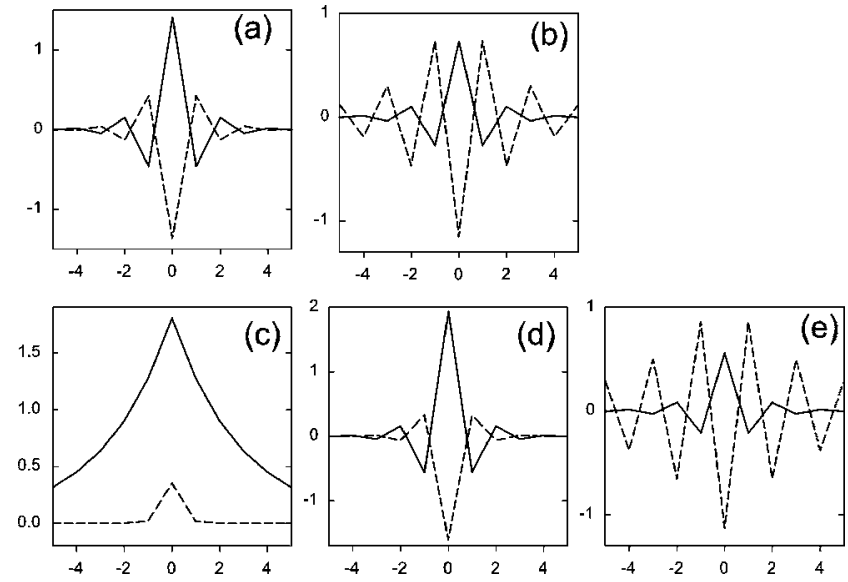

FIG. 2. Same as in Fig. 1 but for $\Delta=5$ and two values of the total power above the threshold: (a), (b) $P=7$ and (c)-(e) $P=10$. In this case, two staggered localized modes appear about the threshold power, and then additional unstaggered mode appears for larger values of $P$.

are stable, and they do not display any bistability.

\section{RESONANT TRANSMISSION}

The two-color localized modes described above can be excited and generated experimentally in arrays of weakly coupled quadratic waveguides being detected through the specific features of the transmission coefficient [12]. Therefore, here we analyze the scattering of a plane FF wave by a quadratic defect waveguide. When the phase-matching conditions are satisfied, after the interaction with the quadratic waveguide, the FF wave generates a SH wave which could either propagate or get trapped being guided by the defect waveguide in the form of a localized mode.

Propagating SH field. To calculate the transmission coefficient in the case of the propagating SH field, we present the fields as

$$
\begin{gathered}
u_{n}(z)=e^{i \lambda_{u^{z}} z} \begin{cases}a e^{i k n}+b_{1} e^{-i k n}, & n<0, \\
c_{1} e^{i k n}, & n \geqslant 0,\end{cases} \\
v_{n}(z)=e^{i \lambda_{v} z} \begin{cases}b_{2} e^{-i q n}, & n<0, \\
c_{2} e^{i q n}, & n \geqslant 0,\end{cases}
\end{gathered}
$$

where $a$ is the amplitude of the FF wave before the scattering, $b_{1,2}$ and $c_{1,2}$ are the amplitudes of the reflected and transmitted FF and SH waves, respectively, and the wave numbers $k$ and $q$ are defined in the domain $0<k, q<\pi$. Using these expressions far from the defect site $n=0$, from the phase-matching condition $\lambda_{v}=2 \lambda_{u}$ we obtain

$$
c_{v} \cos q-\frac{\Delta}{2}=2 c_{u} \cos k .
$$

Equation (12) defines $q=q(k)$ which is real and positive in some interval, $k_{\min }<k<k_{\max }$, where

$$
k_{\min , \max }=\cos ^{-1}\left(\frac{\Delta \pm 2 c_{v}}{4 c_{u}}\right) .
$$

Outside this interval, $q$ is purely imaginary, and this case corresponds to the SH field localized at the waveguide.

Evaluating the fields at the sites $n=-1,0$, we obtain

$$
\begin{gathered}
\lambda_{u} c_{1}=c_{u}\left(c_{1} e^{i k}+a e^{-i k}+b_{1} e^{i k}\right)+2 c_{1}^{*} c_{2}, \\
\lambda_{v} c_{2}=c_{v}\left(c_{2} e^{i q}+b_{2} e^{i q}\right)-c_{2} \Delta+c_{1}^{2}, \\
c_{1}=a+b_{1}, \quad b_{2}=c_{2},
\end{gathered}
$$

the latter condition implies that the SH fields are generated symmetrically. Using these results, we find the important relation $c_{2}=\left(i / 2 c_{v} \sin q\right) c_{1}^{2}$ that allows us to obtain the nonlinear equation for the transmission coefficient of the FF wave, defined as $t(k)=\left|c_{1}\right|^{2} /|a|^{2}$,

$$
t(k)=\frac{1}{[1+B(k) t(k)]^{2}},
$$

where $B(k) \equiv|a|^{2} A(k)=|a|^{2} /\left\{2 c_{u} c_{v} \sin k \sin q(k)\right\}$. By rewriting Eq. (15) in the form

$$
A^{2}(k) t^{3}+2 B(k) t^{2}+t=1,
$$

and using the fact that $A(k)>0$, it is easy to see that this result does not predict bistability. As a matter of fact, the only real and positive solution of Eq. (15) can be found in an analytical form from Eq. (16).

Localized SH field. To calculate the transmission coefficient in the case of the localized SH field, we present the SH field as

$$
v_{n}=e^{i \lambda_{v} z} \begin{cases}b_{2} e^{q n}, & n \leqslant 0, \\ c_{2} e^{-q n}, & n \geqslant 0,\end{cases}
$$

which can be obtained by replacing $q \rightarrow i q$ in Eqs. (11). Real and positive values of $q$ can be found from Eq. (12) that now takes the form

$$
c_{v} \cosh q-\frac{\Delta}{2}=2 c_{u} \cos k .
$$

Wave number $k$ of the incident FF field should be taken outside of the domain $\left(k_{\min }, k_{\max }\right)$, i.e., for $0<k<k_{\min }$ and $k_{\max }<k<\pi$. The relation between the transmitted amplitudes now becomes $c_{2}=c_{1}^{2} /\left(2 c_{v} \sinh q\right)$, and the transmission coefficient $t(k)$ is defined by the equation

$$
t(k)=\frac{1}{1+D^{2}(k) t^{2}(k)},
$$

where $D(k) \equiv|a|^{2} /\left[2 c_{v} c_{u} \sin k \sinh q(k)\right]$. Equation (19) possesses a real and positive solution for $t(k)$ that can be found in an analytic form.

Figures 3(a)-3(d) show some examples of the dependence of the transmission coefficient $t(k)$ on the wave number $k(0<k<\pi)$ for different values of the coupling coefficients $c_{u}, c_{v}$ and the mismatch parameter $\Delta$. In a sharp contrast with the problem of the cubic defect analyzed earlier in Refs. [13] 

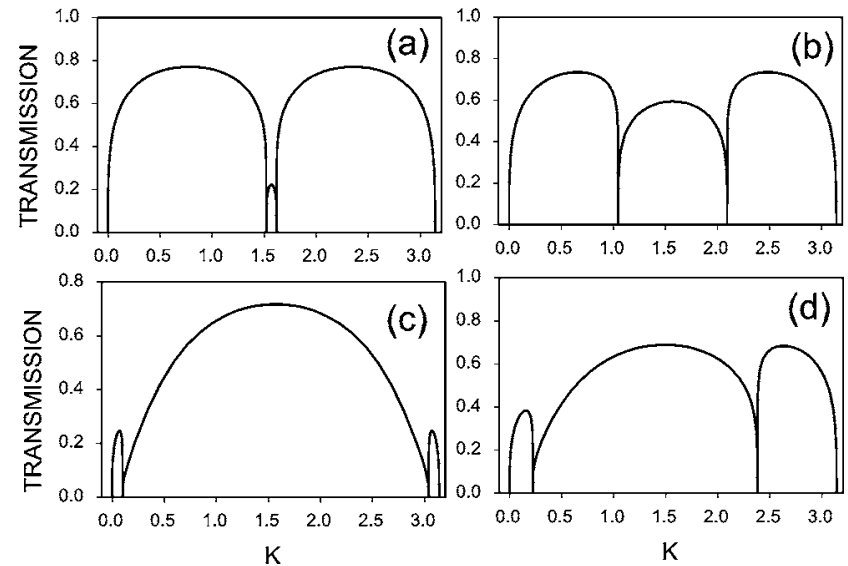

FIG. 3. Transmission coefficient of a plane wave (shown for the fundamental frequency only) after scattering by a nonlinear quadratic defect, for $\Delta=0$ and (a) $c_{v}=1, c_{u}=0.1$, (b) $c_{v}=c_{u}=1$, and (c) $c_{v}=1, c_{u}=1.99$. The case (d) show the asymmetric dependence for $\Delta=0.5$ and $c_{v}=1, c_{u}=1.7$.

where the transmission coefficient does not show any interesting structure and possesses a single maximum only, here the transmission coefficient shows two minima associated with the resonant suppression of the transmission. For small values of the ratio $c_{v} / c_{u}$, the middle interval is narrow and the transmission remain low. The effect of the nonzero mismatch parameter [see Fig. 3(d)] is to shift the position of the middle interval making the transmission curve asymmetric.

The resonant suppression of transmission at some points, i.e., $t\left(k_{\min }\right)=t\left(k_{\max }\right)=0$, corresponds to a novel type of the well-known Fano resonance [14] as recently discussed in
Ref. [12] for a simpler model. Indeed, destructive interference and resonant suppression of transmission is observed when there exists an extra localized state coupled to the propagating mode with the energy inside the linear spectrum. Indeed, the values $q\left(k_{\min }\right)=0$ and $q\left(k_{\max }\right)=\pi$ define the band edges of the propagation spectrum of the SH field, and such resonances take place when the $\mathrm{SH}$ field is generated.

\section{CONCLUSIONS}

Being driven by the recent successful experimental demonstrations of discrete optical solitons with two frequency components mutually locked by a quadratic nonlinearity, in this paper we have studied two-color localized modes in arrays of channel waveguides. We have assumed that the tunable quadratic nonlinearity is introduced as a nonlinear defect by periodic poling of a single waveguide in the array, and we have analyzed the structure and stability of discrete localized modes created by mutual locking of two frequency components. We have shown that, depending on the value of the phase mismatch and the input power, such two-color defect modes can be realized in the same array in three different localized states, and we have studied also the resonant light scattering in the array with the defect waveguide drawing analogies with the Fano resonance.

\section{ACKNOWLEDGMENTS}

The authors thank Andrey Miroshnichenko for useful discussions and collaborations. This work was partially supported by Conicyt and Fondecyt Grants Nos. 1020139 and 7020139 in Chile, and by the Australian Research Council in Australia.
[1] Yu. S. Kivshar and G. P. Agrawal, Optical Solitons: From Fibers to Photonics Crystals (Academic Press, San Diego, 2003), p. 540, and references therein.

[2] D. N. Christodoulides and R. I. Joseph, Opt. Lett. 13, 794 (1988).

[3] H. S. Eisenberg, Y. Silberberg, R. Morandotti, A. R. Boyd, and J. S. Aitchison, Phys. Rev. Lett. 81, 3383 (1998).

[4] A. A. Sukhorukov, Yu. S. Kivshar, H. S. Eisenberg, and Y. Silberberg, IEEE J. Quantum Electron. 39, 31 (2003).

[5] D. N. Christodoulides, F. Lederer, and Y. Silberberg, Nature (London) 424, 817 (2003).

[6] For a review, see A. V. Buryak, P. Di Trapani, D. V. Skryabin, and S. Trillo, Phys. Rep. 370, 63 (2002).

[7] R. Iwanow, R. Schiek, G. I. Stegeman, T. Pertsch, F. Lederer, Y. Min, and W. Sohler, Phys. Rev. Lett. 93, 113902 (2004).

[8] T. Peschel, U. Peschel, and F. Lederer, Phys. Rev. E 57, 1127
(1998).

[9] R. Morandotti, H. S. Eisenberg, D. Mandelik, Y. Silberberg, D. Modotto, M. Sorel, C. R. Stanley, and J. S. Aitchison, Opt. Lett. 28, 834 (2003).

[10] C. Balslev Clausen and L. Torner, Phys. Rev. Lett. 81, 790 (1998).

[11] A. A. Sukhorukov, Yu. S. Kivshar, and O. Bang, Phys. Rev. E 60, R41 (1999).

[12] A. E. Miroshnichenko, Yu. S. Kivshar, R. A. Vicencio, and M. I. Molina, Opt. Lett. 30, 872 (2005).

[13] M. I. Molina and G. P. Tsironis, Phys. Rev. B 47, 15330 (1993); G. P. Tsironis, M. I. Molina, and D. Hennig, Phys. Rev. E 50, 2365 (1994).

[14] S. Flach, A. E. Miroshnichenko, V. Fleurov, and M. V. Fistul, Phys. Rev. Lett. 90, 084101 (2003). 